\title{
Interventional Study Protocol Version
}

National Cancer Institute

\section{Source}

National Cancer Institute. Interventional Study Protocol Version. NCI Thesaurus. Code C93486.

An action plan of a pre-clinical or clinical study in which individuals are assigned by an investigator based on a protocol to receive specific interventions. Study subjects may receive diagnostic, therapeutic or other types of interventions. The assignment of the intervention may or may not be random. The individuals are then followed and biomedical and/or health outcomes are assessed. 